\title{
Alterations in KLRB1 gene expression and a Scandinavian multiple sclerosis association study of the KLRB1 SNP rs4763655
}

\author{
Helle Bach Søndergaard ${ }^{\star, 1}$, Finn Sellebjerg ${ }^{1}$, Jan Hillert $^{2}$, Tomas Olsson ${ }^{3}$, Ingrid Kockum ${ }^{3}$, Magdalena Lindén ${ }^{3}$, \\ Inger-Lise Mero ${ }^{4,5}$, Kjell-Morten Myhr ${ }^{6,7}$, Elisabeth G Celius ${ }^{4}$, Hanne F Harbo ${ }^{4,8}$, Jeppe Romme Christensen ${ }^{1}$, \\ Lars Börnsen ${ }^{1}$, Per Soelberg Sørensen ${ }^{1}$ and Annette Bang Oturai ${ }^{1}$
}

\begin{abstract}
Multiple sclerosis (MS) is a complex autoimmune disease affecting genetically susceptible individuals. A genome-wide association study performed by the International MS Genetics Consortium identified several putative susceptibility genes; among these, the KLRB1 gene is represented by the single-nucleotide polymorphism rs 4763655 . We could confirm a marginally significant association between rs4763655 and MS $(P=0.046$, odds ratio=1.06 (1.00-1.13)) in a large Scandinavian case-control study of $5367 \mathrm{MS}$ patients and 4485 controls. The expression of KLRB1 in blood from MS patients was higher compared with healthy controls $(P<0.001)$, and the KLRB1 expression decreased significantly $(P<0.001)$ after interferon (IFN)- $\beta$ treatment. KLRB1 was expressed in T and natural killer (NK) cells, and expression mainly decreased in NK cells in patients treated with IFN- $\beta$. Collectively, our results indicate that KLRB1 gene expression is altered in MS and likely to be involved in the pathogenesis of the disease, whereas rs4763655 in KLRB1 seems to have a minimal role in MS susceptibility. European Journal of Human Genetics (2011) 19, 1100-1103; doi:10.1038/ejhg.2011.88; published online 25 May 2011
\end{abstract}

Keywords: multiple sclerosis; KLRB1; association study; CD161; case-control study

\section{INTRODUCTION}

Multiple sclerosis (MS) is a chronic, inflammatory disease that causes degradation of myelin sheaths and destruction of axons in the central nervous system. The aetiology of MS is not yet fully understood, but individuals that are genetically susceptible are thought to be triggered to develop MS by environmental factors, of which only a few have been identified, such as vitamin D status, infectious mononucleosis (Epstein-Barr virus) and smoking. ${ }^{1,2}$

Recently, genome-wide association studies (GWASs) have identified several gene loci associated with MS corroborated by large replication and meta-analyses, as recently reviewed. ${ }^{3}$ Despite the overall minor contribution from each single-nucleotide polymorphism (SNP), more than 12 gene loci are today identified at the genome-wide significance level $\left(P<5 \times 10^{-7}\right){ }^{3}$ Thus, besides the well-known large contribution of the class II region of the major histocompatibility complex to the risk at developing $\mathrm{MS},{ }^{4}$ there is increasing evidence of a network of several gene loci with minor predisposing effects. ${ }^{5}$

In the GWAS performed by the International Multiple Sclerosis Genetic Consortium, a polymorphism (rs4763655) in the killer cell lectin-like receptor, subfamily $\mathrm{B}$, member 1 (KLRB1) was found associated with MS and among the top 17 list of genes identified $\left(P=6.85 \times 10^{-4}\right.$, odds ratio $\left.(\mathrm{OR})=1.10(1.04-1.17)\right) .^{6}$ Thus, the associated KLRB1 gene SNP may be part of the network of genes, with minor contributions to the development of MS. The KLRB1 gene variant is located in intron 1 of the KLRB1 gene that encodes the CD161/NKR-P1A protein, a C-type lectin receptor expressed on the surface of natural killer (NK) cells and subtypes of T lymphocytes. ${ }^{7}$ Importantly, CD161 is expressed on the surface of CD4 ${ }^{+} \mathrm{T}$-helper cells producing interleukin 17 (Th17 cells) that are involved in the pathogenesis in $\mathrm{MS}^{8}$ and on regulatory NK cells (reviewed by Vivier et $a l^{9}$ ). The KLRB1 gene is located on chromosome 12p12-13, and in humans, it is found as a single homologue. ${ }^{7} \mathrm{CD} 161$ binds to lectin-like transcript-1, expressed on activated antigen-presenting cells, ${ }^{10}$ which is found to elicit an inhibitory response on NK cell cytotoxicity. ${ }^{11,12}$ Whether CD161 has a co-stimulatory effect on T-cells, as previously suggested, ${ }^{11}$ is still being debated. ${ }^{10}$

In this study, we attempted to replicate the KLRB1 SNP association with MS in a Scandinavian population. Furthermore, we compared KLRB1 gene expression in 39 healthy controls with that in 39 untreated and 33 interferon (IFN)- $\beta$-treated Danish MS patients, and studied the relationship between KLRB1 genotype and disease course in more than 600 IFN- $\beta$-treated Danish MS patients.

\section{SUBJECTS AND METHODS}

\section{Subjects}

All included Scandinavian MS patients fulfilled the revised McDonald criteria for definite MS from $2005^{13}$ and were randomly recruited by neurologists from MS centres. Informed consent was obtained from all participants, and the local

\footnotetext{
${ }^{1}$ Department of Neurology, Danish Multiple Sclerosis Center, University Hospital Rigshospitalet, Copenhagen, Denmark; ${ }^{2}$ Multiple Sclerosis Research Group, Department of Clinical Neuroscience, Centre for Molecular Medicine, Karolinska University Hospital, Stockholm, Sweden; ${ }^{3}$ Neuroimmunology Unit, Department of Clinical Neurosciences, Centre for Molecular Medicine, Karolinska University Hospital, Stockholm, Sweden; ${ }^{4}$ Department of Neurology, Oslo University Hospital, Oslo, Norway; ${ }^{5}$ Institute of Immunology, Oslo University Hospital, Oslo, Norway; ${ }^{6}$ Norwegian Multiple Sclerosis Registry and Biobank, Department of Neurology, Haukeland University Hospital, Bergen, Norway; ${ }^{7}$ Department of Clinical Medicine, University of Bergen, Bergen, Norway; ${ }^{8}$ Department of Neurology, University of Oslo, Oslo, Norway

*Correspondence: Dr HB Søndergaard, Department of Neurology, Danish Multiple Sclerosis Center, University Hospital Rigshospitalet, Blegdamsvej 9 , DK-2100 Copenhagen, Denmark. Tel: +45 35456707; Fax: +45 35456316; E-mail: hbs@rh.dk
}

Received 13 August 2010; revised 7 April 2011; accepted 16 April 2011; published online 25 May 2011 
ethics board has approved the experimental protocols. Owing to recruitment criteria, all included patients and controls were Caucasians. Danish individuals were recruited from the Danish MS Center in Copenhagen, and controls consisted of healthy hospital staff members for the large majority of consecutive blood donor controls from Copenhagen University Hospital. Norwegian patients were recruited from neurological clinics in the Oslo area or from the national MS register and Biobank. The Norwegian control samples were healthy blood donors recruited through the Norwegian Bone Marrow Donor Registry (http://www.nordonor.org/). Swedish patients were recruited from neurological clinics in the Stockholm area or were part of a nation-wide study (EIMS). ${ }^{1}$ The Swedish controls were either healthy blood donors or population-based controls matched to newly diagnosed MS patients in the EIMS Study. For a detailed description of the cohort of untreated and IFN- $\beta$-treated MS patients used for gene expression analysis, please see Krakauer et al (2008). ${ }^{14}$ Allele-specific gene expression was investigated in 129 Caucasian healthy control subjects sampled in 2004 among healthy staff personals and is part of the healthy controls used for genotyping (mean age (SD) 44.6 years (13.6), gender ratio 1.9).

\section{Molecular genetic analysis}

Genotyping of the KLRB1 SNP rs4763655 was performed on all Scandinavian individuals using TaqMan allelic discrimination. Predesigned primers and probes were obtained from Applied Biosystems (Foster City, CA, USA), and genotyping protocols were followed as described by the manufacturer (Applied Biosystems Inc.). PCR and end-point scoring were performed with a 7500 realtime PCR system. Genotype detection threshold was set at $90 \%$. Genotype accuracy was determined on $25 \%$ of plates from the Danish cohort (39 replicate samples), $5 \%$ of plates from the Norwegian cohort (2 replicate samples) and $100 \%$ of plates from the Swedish cohort (42 CEPH (Centre d'etude du polymorphisme humain) DNA samples that were replicated, at least 3 different DNA samples on each 96-well plate). In addition, 49 Danish and 33 Swedish samples were analysed on two separate days. All intra- and inter-assay replicates showed $100 \%$ genotype concordance. The CEPH DNA samples had the same genotype as reported on the HapMap home page.

\section{RNA isolation and expression analysis}

Samples from MS patients treated with IFN- $\beta$ (Avonex, Biogen Idec, Hilleroed, Denmark) were taken 9-12 h after injection. RNA was extracted from whole blood collected in PAXgene tubes (QIAGEN, Copenhagen, Denmark) using the RNeasy Plus kit (QIAGEN) and reverse transcribed using the High Capacity cDNA RT kit (Applied Biosystems). Real-time PCR was then performed on diluted cDNA template with assay-specific primers and probes (KLRB1: Hs00174469_ml and GAPDH: Hs99999905_ml) using TaqMan technology, and PCR amplifications were performed using a 7500 real-time PCR System
(Applied Biosystems). An expression index was calculated by the $2^{-\Delta \Delta \mathrm{Ct}}$ method for relative quantification, ${ }^{15}$ where data were normalised to the reference gene GAPDH, and pooled total RNA from 50 healthy subjects was used as a calibrator. Log-transformed index values were analysed by an unpaired t-test. Kruskal-Wallis test was used for analysis of allele-specific gene expression (Supplementary Figure 1A and B).

\section{Statistical analysis}

Cohorts from Sweden, Norway and Denmark were tested for heterogeneity by the Breslow-Day test $\left(\chi^{2}=2.011, P=0.37\right)$ before combined analyses. For the combined analysis, Cochran-Mantel-Haenszel $\chi^{2}$-test was used. Comparison of allele frequencies within each cohort was performed using PLINK v.1.07 (http:// pngu.mgh.harvard.edu/purcell/plink/). Genotypes of KLRB1 rs4763655 SNP were analysed by a Kaplan-Meier analysis in relation to the clinical parameters progression $(n=618)$ and relapse $(n=608)$ of IFN- $\beta$-treated MS patients. Test of equality between genotypes and clinical parameters were performed with Mantel-Cox analysis.

\section{RESULTS AND DISCUSSION}

We performed a replication study typing the KLRB1 SNP rs4763655 in a Scandinavian population comprising 5367 MS cases and 4485 controls from Norway, Sweden and Denmark. We limited our analyses to Scandinavian populations, as these populations are genetically homogenous and, therefore, well suited to look for small genetic effects. ${ }^{16}$ Power calculations using Quanto v.1.2 (http://hydra.usc.edu/ gxe/) demonstrated more than $80 \%$ power to replicate the MS association of rs4763655 at a significance level of 0.05 , with an OR set at 1.1. Controls from the three study populations were tested for deviation from Hardy-Weinberg equilibrium, and none showed significant deviation $(P>0.25)$. The overall KLRB1 rs4763655 SNP genotyping efficiency was $>98 \%$. Clinical characteristics for the three populations are shown in Supplementary Table 1. The risk allele frequency (A allele) was high in MS cases in all the three populations (Table 1); however, only the Danish cohort showed a trend towards a significant association $(P=0.05)$. The homogeneity of the odds ORs from the Scandinavian populations was tested by the Breslow-Day test excluding significant heterogeneity $(P=0.4)$. This allowed for a combined Scandinavian analysis performed by the Cochran-MantelHaenszel test using PLINK v1.07. ${ }^{17}$ The rs4763655 association with MS was replicated in the combined Scandinavian case-control analysis with nominal significance $(P=0.046, \mathrm{OR}=1.06(1.00-1.13)$; Table 1$)$.

Table 1 Scandinavian MS case-control association analysis of KLRB1 SNP, rs4763655

\begin{tabular}{|c|c|c|c|c|c|c|c|c|c|}
\hline Population & $\mathrm{N}$ & $A A$ & $A G$ & $G G$ & RAF cases & RAF control & $\chi^{2}$ & $\mathrm{P}$-value & OR $(95 \% \mathrm{Cl})$ \\
\hline \multicolumn{10}{|l|}{ Norwegian } \\
\hline Cases & 1903 & 224 & 866 & 813 & & & & & \\
\hline Controls & 1540 & 178 & 702 & 660 & 0.35 & 0.34 & 0.023 & 0.88 & $1.01(0.91-1.11)$ \\
\hline \multicolumn{10}{|l|}{ Swedish } \\
\hline Cases & 2246 & 273 & 1019 & 954 & & & & & \\
\hline Controls & 1820 & 193 & 821 & 806 & 0.35 & 0.33 & 2.21 & 0.14 & $1.07(0.98-1.18)$ \\
\hline \multicolumn{10}{|l|}{ Danish } \\
\hline Cases & 1218 & 159 & 584 & 475 & & & & & \\
\hline Controls & 1125 & 141 & 490 & 494 & 0.37 & 0.34 & 3.76 & 0.053 & $1.13(1.00-1.28)$ \\
\hline Combined analysis & $\mathrm{N}$ & $A A$ & $A G$ & $G G$ & & & $\chi^{2}$ & $\mathrm{P}$-value & OR $(95 \% \mathrm{Cl})$ \\
\hline \multicolumn{10}{|c|}{ Scandinavian combined } \\
\hline Cases & 5367 & 654 & 2468 & 2245 & & & 3.978 & 0.046 & $1.06(1.001-1.13)$ \\
\hline Controls & 4485 & 512 & 2013 & 1960 & & & & & \\
\hline
\end{tabular}

Abbreviations: OR, odds ratio; RAF, risk allele frequency. 
All replicate samples showed $100 \%$ genotype concordance and clear separation between genotype groups. However, not all plates contained replicates, and it cannot be entirely ruled out that genotyping errors might have occurred. Indeed, as the significance of the association was only marginal, it is still possible that variation in the distribution of genotypes in the different groups, either by chance or due to genotyping errors, might have influenced the results.

KLRB1 gene expression was measured in whole blood from 33 treated and 39 untreated Danish MS patients, and we observed a 2.1-fold higher expression in blood cells from relapsing-remitting MS patients compared with 39 healthy controls $\left(P=4.1 \times 10^{-6}\right.$; Figure 1$)$. Furthermore, MS patients treated with IFN- $\beta$ for more than 6 months had 3.8-fold lower expression than untreated MS patients $\left(P=2.2 \times 10^{-12}\right.$; Figure 1$) ; P$-values are Bonferroni corrected, as more targets were investigated in parallel studies. ${ }^{18}$

Bioinformatic analyses have suggested that rs4763655 may be located in a transcription factor site (analysis performed by using http://www.genomatix.de). If the KLRB1 risk allele has an effect on the expression levels of $K L R B 1$, we would anticipate seeing a difference in expression depending on KLRB1 genotype. Thus, we investigated KLRB1 gene expression and rs4763655 SNP genotypes in blood mononuclear cells from 129 healthy controls. KLRB1 expression was lowest in subjects with the AA genotype, but we did not observe significant differences in gene expression between the AA, AG and GG genotypes (Kruskal-Wallis test, $P=0.179$, see Supplementary Figure 1A). Furthermore, comparing KLRB1 gene expression in rs4763655 SNP genotype groups in $34 \mathrm{MS}$ cases did not show any statistically significant differences either (Kruskal-Wallis test, $P=0.861$, see Supplementary Figure 1B).

We then investigated KLRB1 expression in immunomagnetically isolated sub-populations of mononuclear blood cells from untreated and IFN- $\beta$-treated Danish MS patients (number of patients, $n=4$ ). A substantial proportion of KLRB1 mRNA was derived from NK cells (65\% in healthy subjects (see Supplementary Figure 2), and IFN- $\beta$ treatment reduced KLRB1 gene expression mainly in the NK cell population. These data indicate that CD161, the KLRB1 gene product, in MS patients may exert its function in NK cells, in addition to the $\mathrm{CD}^{+}$Th17 cells, previously reported to express CD161. ${ }^{8}$ Previous studies have shown that untreated MS patients ${ }^{19}$ and MS patients treated with

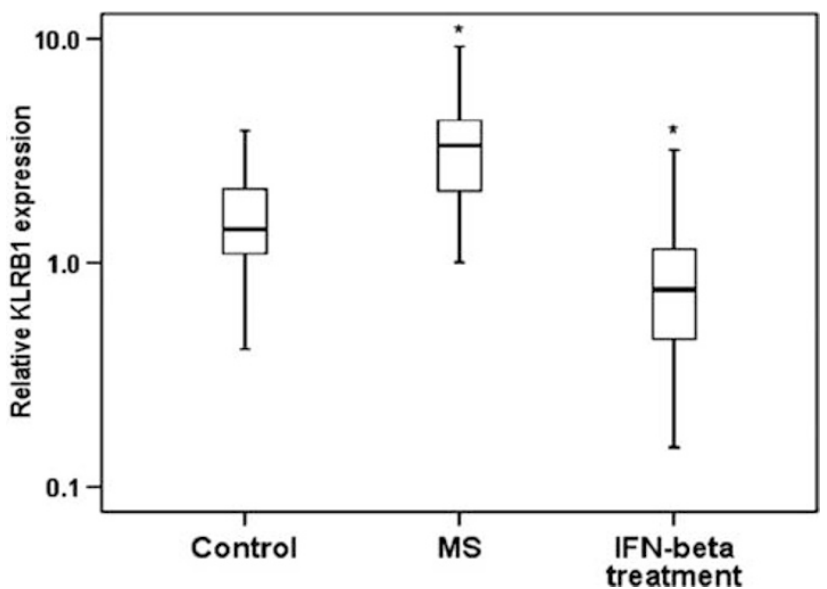

Figure 1 Relative gene expression of $K L R B 1$ in MS patients and healthy controls. Box plots showing higher relative KLRB1 expression in $39 \mathrm{MS}$ patients compared with 39 healthy controls $\left({ }^{*} P<0.001\right)$ and relative lower $K L R B 1$ expression in 33 IFN- $\beta$-treated $\mathrm{MS}$ patients compared with untreated MS patients $\left({ }^{*} P<0.001\right)$. qPCR analysis was performed on MS patients and age- and gender-matched healthy controls.
IFN- $\beta^{20}$ have a lower percentage of circulating NK cells, and other studies suggest that natural $\mathrm{NK}$ cells ${ }^{21}$ and regulatory NK cells induced by treatment with daclizumab can have regulatory functions in $\mathrm{MS}^{22}$

It is uncertain whether the decrease in KLRB1 expression in NK cells from MS patients treated with IFN- $\beta$ represents repression of gene expression directly by IFN- $\beta$, an indirect effect by changes in differential cytokine profiles, ${ }^{23}$ or a simple decrease in the percentage of NK cell sub-populations that express CD161.

The pronounced effect of IFN- $\beta$ treatment on gene expression led us to investigate the potential effect of KLRB1 rs4763655 genotypes on disease activity and progression in both untreated Danish MS patients and in 620 prospectively collected Danish MS patients treated with IFN- $\beta$ (clinical data are shown in Supplementary Table 2). In untreated patients, KLRB1 gene expression did not correlate with disease activity as assessed by magnetic resonance imaging with gadolinium contrast for detection of active lesions (data not shown). In IFN- $\beta$-treated patients, the KLRB1 genotype had no effect on disease activity, measured as gadolinium-enhancing lesions, on time to first relapse (Mantel-Cox $P=0.92$ ) or on time to progression in EDSS in a KaplanMeier survival plot (Mantel-Cox $P=0.83$; Figures 2a and $b$ ).
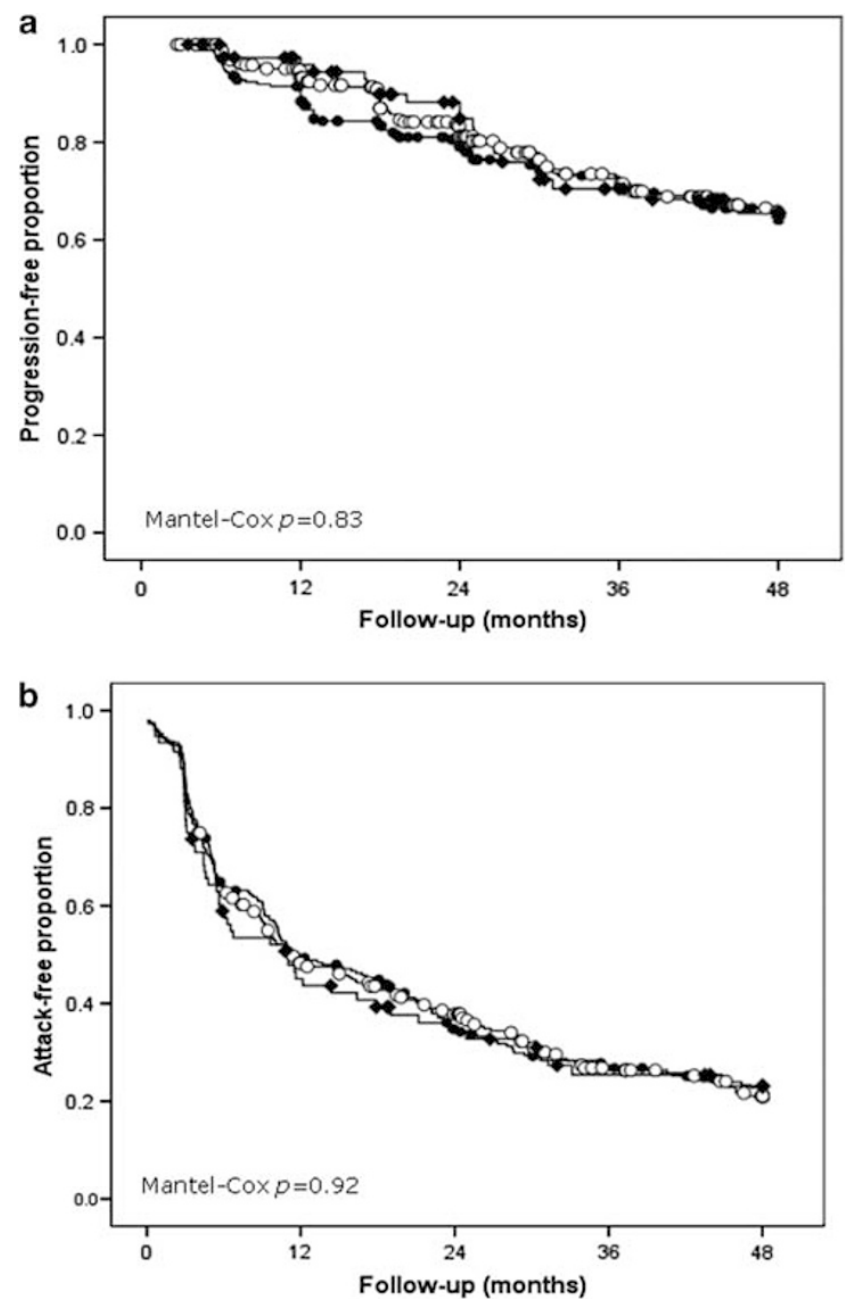

Figure 2 Survival plots showing the relation between the $K L R B 1$ rs4763655 genotype and the clinical parameters' progression and relapse. (a) The $K L R B 1$ genotype does not have an effect on progression among IFN$\beta$-treated patients. (b) The $K L R B 1$ genotype does not change the time to first relapse among IFN- $\beta$-treated patients. AA, rhombi; $A G$, open circles; GG, closed circles. 
In conclusion, our findings demonstrate that the KLRB1 rs4763655 SNP is marginally associated with MS in a large combined Scandinavian analysis. Even with $\sim 5000 \mathrm{MS}$ patients and controls included, this study had limited power. We cannot exclude that another genetic variant in the vicinity of the rs4763655 SNP might be the true diseaseassociated variant in this region. However, an LD plot generated from HapMap CEU data covering a region on chromosome 12 from 9.4 to 9.75 Mb did not show any LD to the nearest genes (Supplementary Figure $3 \mathrm{~A}$ and B). Expression of KLRB1 in healthy subjects was not significantly affected by the SNP, and the risk genotype did not influence clinical parameters after IFN- $\beta$ treatment. Any direct evidence for a causal connection between the marginal association and KLRB1 gene expression levels was not observed. However, we identified higher expression of KLRB1 in blood from MS patients compared with healthy controls and found that KLRB1 expression decreased significantly after IFN- $\beta$ treatment. KLRB1 was expressed in $\mathrm{T}$ and NK cells, and expression mainly decreased in NK cells in patients treated with IFN- $\beta$. Indeed, the observed increased expression of KLRB1 in MS patients, together with the substantial decrease observed in patients treated with IFN- $\beta$, indicates that the KLRB1 gene product may be of importance in MS, possibly as a treatment target.

\section{CONFLICT OF INTEREST}

HBS, ML, JRC, EGC, IK, ILM, HFH and LB declare no conflict of interest. $\mathrm{JH}$ has received unrestricted research support from Biogen Idec, MercSerono and Bayer-Schering. TO has received unrestricted research support from Biogen Idec, Merck Serono, Sanofi-Aventis and Bayer Shering. KMM has received honoraria for lecturing and travel expenses for attending meetings, and research support from Biogen Idec, Bayer, Merck Serono or Sanofi-Aventis. FS has received honoraria for consulting or lecturing, travel grants or research grants from Bayer-Schering, Biogen Idec, Merck Serono, Novo Nordisk, SanofiAventis, Schering-Plough and Teva; PSS has received funding, honoraria for consulting or lecturing or research grants from Baxter, BayerSchering, Biogen Idec, BioMS Medical, Merck Serono, Novartis, Sanofi-Aventis and Teva. ABO received travel grants and speakers honoraria from Biogen Idec, Bayer-Schering Pharma and Merck Serono.

\section{ACKNOWLEDGEMENTS}

We thank Joy Mendel-Hartvig, Vibeke Fuglholt and Marie Koefoed for excellent laboratory work. The Danish MS Society, the Warwara Larsen Foundation, the Johnsen Foundation and The Danish Council for Strategic Research supported the Danish part of the study. The Research Council of Norway and South Eastern Norway Regional Health Authority supported the Norwegian part of the study. We thank the Norwegian Bone Marrow Donor Registry for collaboration in establishment of the Norwegian control material, and we acknowledge the Norwegian MS Register and Biobank for contribution of a proportion of the MS samples. Swedish Medical Research Council financed the Swedish part of the project (sample collection). Grants were provided by the Swedish Council for Working Life and Social Research, the fp6 EU program Neuropromise (LSHM-CT-2005-018637), Bibbi and Niels Jensens Foundation, Montel Williams Foundation, the Söderberg foundation and the Swedish Association for Persons with Neurological Disabilities.

1 Hedstrom AK, Baarnhielm M, Olsson T, Alfredsson L: Tobacco smoking, but not Swedish snuff use, increases the risk of multiple sclerosis. Neurology 2009; 73: 696-701.

2 Ebers GC: Environmental factors and multiple sclerosis. Lancet Neurol 2008; 7 : 268-277.

3 Oksenberg JR, Baranzini SE: Multiple sclerosis genetics-is the glass half full, or half empty? Nat Rev Neurol 2010; 6: 429-437.

4 Jersild C, Fog T, Svejgaard A: HI-A antigens and multiple-sclerosis. Lancet 1972; 1: 1240.

5 Bush WS, Sawcer SJ, De Jager PL et al: Evidence for polygenic susceptibility to multiple sclerosis-the shape of things to come. Am J Hum Genet 2010; 86: 621-625.

6 Hafler DA, Compston A, Sawcer S et al: Risk alleles for multiple sclerosis identified by a genomewide study. N Engl J Med 2007; 357: 851-862.

7 Lanier LL, Chang C, Phillips JH: Human NKR-P1A. A disulfide-linked homodimer of the C-type lectin superfamily expressed by a subset of NK and T lymphocytes. J Immunol 1994; 153: 2417-2428.

8 Cosmi L, De Palma R, Santarlasci V et al: Human interleukin 17-producing cells originate from a CD161(+)CD4(+) T cell precursor. J Exp Med 2008; 205: 1903-1916.

9 Vivier E, Tomasello E, Baratin M, Walzer T, Ugolini S: Functions of natural killer cells. Nat Immunol 2008; 9: 503-510.

10 Rosen DB, Cao W, Avery DT et al: Functional consequences of interactions between human NKR-P1A and its ligand LLT1 expressed on activated dendritic cells and B cells. J Immunol 2008; 180: 6508-6517.

11 Aldemir H, Prod'homme V, Dumaurier MJ et al: Cutting edge: lectin-like transcript 1 is a ligand for the CD161 receptor. J Immunol 2005; 175: 7791-7795.

12 Rosen DB, Bettadapura J, Alsharifi M, Mathew PA, Warren HS, Lanier LL: Cutting edge: lectin-like transcript-1 is a ligand for the inhibitory human NKR-P1A receptor. $\mathrm{J}$ Immunol 2005; 175: 7796-7799.

13 Polman CH, Reingold SC, Edan G et al: Diagnostic criteria for multiple sclerosis: 2005 revisions to the 'McDonald Criteria'. Ann Neurol 2005; 58: 840-846.

14 Krakauer M, Sorensen P, Khademi M, Olsson T, Sellebjerg F: Increased IL-10 mRNA and IL-23 mRNA expression in multiple sclerosis: interferon-beta treatment increases IL-10 mRNA expression while reducing IL-23 mRNA expression. Mult Scler 2008; 14: $622-630$.

15 Livak KJ, Schmittgen TD: Analysis of relative gene expression data using real-time quantitative PCR and the 2(T)(-Delta Delta C) method. Methods 2001; 25: 402-408.

16 Novembre J, Johnson T, Bryc K et al: Genes mirror geography within Europe. Nature 2008; 456: 98-101.

17 Purcell S, Neale B, Todd-Brown K et al: PLINK: A tool set for whole-genome association and population-based linkage analyses. Am J Hum Genet 2007; 81: 559-575.

18 Hesse D, Krakauer M, Lund $\mathrm{H}$ et al: Breakthrough disease during interferon-[beta] therapy in MS: no signs of impaired biologic response. Neurology 2010; 74: $1455-1462$.

19 De Jager PL, Rossin E, Pyne S et al: Cytometric profiling in multiple sclerosis uncovers patient population structure and a reduction of CD8(low) cells. Brain 2008; 131: 1701-1711.

20 Sellebjerg F, Ross C, Koch-Henriksen $\mathrm{N}$ et al: CD26+ CD4+ T cell counts and attack risk in interferon-treated multiple sclerosis. Mult Scler 2005; 11: 641-645.

21 Takahashi K, Aranami T, Endoh M, Miyake S, Yamamura T: The regulatory role of natural killer cells in multiple sclerosis. Brain 2004; 127: 1917-1927.

22 Bielekova B, Catalfamo M, Reichert-Scrivner S et al: Regulatory CD56(bright) natural killer cells mediate immunomodulatory effects of IL-2Ralpha-targeted therapy (daclizumab) in multiple sclerosis. Proc Natl Acad Sci USA 2006; 103: 5941-5946.

23 Johansson S, Berg L, Hall H, Hoglund P: NK cells: elusive players in autoimmunity. Trends Immunol 2005; 26: 613-618.

Supplementary Information accompanies the paper on European Journal of Human Genetics website (http://www.nature.com/ejhg) 